$$
b_{1}=b_{2}=\cdots=b_{n}=0, \beta=0, \rho_{2}=0, \epsilon=1 \quad \text { of } \mathrm{V} \text { and VI, }
$$

and

$$
a_{1}=a_{2}=\cdots=a_{n}=0, \alpha=0, \rho=0, e=1, \lambda=1 \quad \text { of VII, }
$$

are due to G. Szegö.

UNIVERSITY OF HUNGARY, SZEGED

\title{
SOME REMARKS ON POLYNOMIALS
}

\section{P. ERDÖS}

This note contains some disconnected remarks on polynomials.

Let $f_{n}(x)=\prod_{i=1}^{n}\left(x-x_{i}\right), \quad-1 \leqq x_{1} \leqq x_{2} \leqq \cdots \leqq x_{n} \leqq 1$. Denote by $-1 \leqq y_{1} \leqq \cdots \leqq y_{n-1} \leqq 1$ the roots of $f_{n}^{\prime}(x)$. We prove the following theorem.

THEOREM 1. For all $n$

$$
\left|f_{n}(-1)\right|+\left|f_{n}(+1)\right|+\sum_{i=1}^{n-1}\left|f_{n}\left(y_{i}\right)\right| \leqq 2^{n} \text {. }
$$

For $n \geqq 3$

$$
\left|f_{n}(-1)\right|^{1 / 2}+\left|f_{n}(+1)\right|^{1 / 2}+\sum_{i=1}^{n-1}\left|f_{n}\left(y_{i}\right)\right|^{1 / 2} \leqq 2^{n / 2} .
$$

For $n \geqq n_{0}(k)$

$$
\left|f_{n}(-1)\right|^{1 / k}+\left|f_{n}(+1)\right|^{1 / k}+\sum_{i=1}^{n-1}\left|f_{n}\left(y_{i}\right)\right|^{1 / k} \leqq 2^{n / k} .
$$

REMARK. If $y_{i}=y_{i+1}$ or $-1=y_{1},+1=y_{n-1}$ the corresponding summands clearly vanish.

Clearly

$$
\begin{gathered}
\left|f_{n}(-1)\right| \leqq \\
\left(1-x_{1}\right) 2^{n-1}, \quad\left|f_{n}\left(y_{i}\right)\right| \leqq\left|y_{i}-x_{i+1}\right| 2^{n-1}, \\
\left|f_{n}(+1)\right| \leqq\left(1-x_{n}\right) 2^{n-1} .
\end{gathered}
$$

Thus

Received by the editors March 28, 1947. 


$$
\begin{aligned}
& \left|f_{n}(-1)\right|+\left|f_{n}(+1)\right|+\sum_{i=1}^{n-1}\left|f_{n}\left(y_{i}\right)\right| \\
& \leqq\left(\left(1-x_{1}\right)+\sum_{i=1}^{n-1}\left(y_{i}-x_{i+1}\right)+\left(1-x_{n}\right)\right) 2^{n-1} \leqq 2^{n},
\end{aligned}
$$

which proves (1)

We have by the inequality of the geometric and arithmetic mean

$$
\begin{aligned}
&\left|f_{n}(-1)\right|^{1 / 2} \leqq \frac{\left(1-x_{1}\right)+\left(1-x_{2}\right)}{2} 2^{n / 2-1}, \\
&\left|f_{n}\left(y_{i}\right)\right|^{1 / 2} \leqq \frac{\left(x_{i+1}-x_{i}\right)}{2} 2^{n / 2-1}, \\
&\left|f_{n}(+1)\right|^{1 / 2} \leqq \frac{\left(1-x_{n}\right)+\left(1-x_{n-1}\right)}{2} 2^{n / 2-1} .
\end{aligned}
$$

Thus we evidently have for $n \geqq 3$

$$
\left|f_{n}(-1)\right|^{1 / 2}+\left|f_{n}(+1)\right|^{1 / 2}+\sum_{i=1}^{n-1}\left|f_{n}\left(y_{i}\right)\right|^{1 / 2} \leqq 2^{n / 2},
$$

which proves (2).

$f_{1}(x)=x$ and $f_{2}(x)=x^{2} / 2-1$ shows that (2) is false for $n<3$. Clearly equality occurs in (1) and (2) only for $\pm(1 \pm X)^{n}$.

The proof of (3) is more complicated and since the proof does not present any particular interest we are just going to sketch it. Let $f_{n}(x)$ be the polynomial which maximizes the sum (3). If (3) is not true we must have

$$
\left|f_{n}\left(x_{0}\right)\right|=\max _{-1 \leqq x \leqq 1}\left|f_{n}(x)\right|>\frac{2^{n}}{n^{k}}
$$

But then it is easy to see that $x_{0}$ does not lie in $(1-\epsilon,-1+\epsilon)$; without loss of generality we can assume that $1-\epsilon<x_{0} \leqq 1$, and $n+o(n)$ of the $x_{i}$ are in $(-1+\delta,-1)$. But then a simple computation shows that $f_{n}(x)$ has no roots in $(1-\epsilon, 1)$ and thus $x_{0}=1$. (This is clear since if we move any possible root of $f_{n}(x)$ in $(1-\epsilon, 1)$ to -1 , we clearly increase the sum (3).) By the same argument we obtain by a simple calculation that all the roots of $f_{n}(x)$ have to be in -1 , which proves (3) and completes the proof of Theorem 1.

At present I can not determine the exact value of $n_{0}(k)$.

Let $g_{n}(z)=\prod_{i=1}^{n}\left(z-z_{i}\right),\left|z_{i}\right|=1$. Denote by $u_{1}, u_{2}, \cdots$ the local extremal points of $g_{n}(z)$, that is, the points where the vector $g_{n}^{\prime}(z)$ 
points either towards the origin or away from it. I conjectured that

$$
\sum_{i}\left|g_{n}\left(u_{i}\right)\right| \leqq 2^{n}
$$

Professor Breusch ${ }^{1}$ proved this conjecture for sufficiently large $n$. The proof is complicated. For small values of $n$ he showed by examples that the result is false.

Let $-1=x_{0}<x_{1} \leqq \cdots \leqq x_{n}=x_{n+1}=1$. Put $\omega(x)=\prod_{i=1}^{n}\left(x-x_{i}\right)$, $l_{k}(x)=\omega(x) / \omega\left(x_{k}\right)\left(x-x_{k}\right)$, the fundamental functions of Lagrange interpolation. The problem of determining the set for which

$$
\max _{-1 \leqq x \leqq 1} \sum_{k=1}^{n}\left|l_{k}(x)\right|
$$

is minimal is still unsolved. It has been conjectured, but never proved, that the minimum is attained for the points for which all the $n+1$ sums

$$
\max _{x_{i} \leqq x \leqq x_{i+1}} \sum_{k=1}^{n}\left|l_{k}(x)\right|, \quad i=0,1, \cdots, n,
$$

are equal. If the $x_{i}$ are the roots of $T_{n}(x)$ (the $n$th Tchebychef polynomial), then a simple computation shows that the sums (4) all equal

$$
\frac{2}{\pi} \log n+O(1)
$$

S. Bernstein ${ }^{2}$ proved that for any $-1 \leqq x_{1} \leqq x_{2} \leqq \cdots \leqq x_{n} \leqq 1$

$$
\max _{-1 \leqq x \leqq 1} \sum_{k=1}^{n}\left|l_{k}(x)\right|>(1+o(1)) \frac{2}{\pi} \log n,
$$

and I proved ${ }^{3}$ that

$$
\left.\max _{-1 \leqq x \leqq 1} \sum_{k=1}^{n}\left|l_{k}(x)\right|>\frac{2}{\pi} \log n-c \quad \text { (c absolute constant }\right) .
$$

We consider a slightly different problem. We prove the following theorem.

THEOREM 2. Let $-1=x_{0} \leqq x_{1} \cdots \leqq x_{n} \leqq x_{n+1}=1$. Then for some $i$

$$
\max _{x_{i<x<x_{i+1}}} \sum_{k=1}^{n}\left|l_{k}(x)\right|<n^{1 / 2} \text {. }
$$

1 Oral communication.

2 Bull. Acad. Sci. URSS. Sér. Math. (1931) pp. 1025-1050.

3 Unpublished. 
REMARK. $n^{1 / 2}$ in (5) can very likely be improved to $c \log n$. In fact it is likely that

$$
\min _{0 \leqq x_{i} \leqq n} \max _{x_{i} \leqq x \leqq x_{i+1}} \sum_{k=1}^{n}\left|l_{k}(x)\right|
$$

assumes its maximum when all the sums (4) are equal.

If, for some $i, x_{i}=x_{i+1}$ then (5) is obvious. Assume that $x_{i} \neq x_{i+1}$, $0 \leqq i \leqq n+1$. Consider the equation $\sum_{k=1}^{n} l_{k}^{2}(x)=1$. The number of solutions is not greater than $2 n-2$ and $x_{1}, x_{2}, \cdots, x_{n}$ are solutions. Thus a simple argument shows that for some $i, 1 \leqq i \leqq n-1$,

$$
\sum_{k=1}^{n} l_{k}^{2}(x)<1 \quad \text { for } x_{i}<x<x_{i+1} .
$$

But then clearly (from Schwartz's inequality)

$$
\sum_{k=1}^{n}\left|l_{k}(x)\right|<n^{1 / 2} \quad \text { for } x_{i} \leqq x \leqq x_{i+1}
$$

which proves Theorem 2.

In one of his interesting papers Schur ${ }^{4}$ proves among others the following result: Let $a_{0} x^{n}+\cdots+a_{n}$ be a polynomial with integer coefficients, all whose roots are in $(-1,+1)$ and are different. Then for sufficiently large $n,\left|a_{0}\right|>\left(2^{1 / 2}-\epsilon\right)^{n}$. We prove the stronger theorem:

THEOREM 3. Let $f_{n}(x)=a_{0} x^{n}+\cdots+a_{n}$ be a polynomial with integer coefficients and $f_{n}(-1) \neq 0, f_{n}(0) \neq 0, f_{n}(+1) \neq 0$. Then, $\left|a_{0}\right| \geqq 2^{n / 2}$.

We have $\left(x_{1}, x_{2}, \cdots, x_{n}\right.$ are the roots of $\left.f_{n}(x)\right)$

$$
\left|f_{n}(-1) f_{n}^{2}(0) f_{n}(+1)\right|=\left|a_{0}^{4} \prod_{i=1}^{n}\left(1-x_{i}^{2}\right) x_{i}^{2}\right| \geqq 1 \text {. }
$$

But $\left|\left(1-x_{i}^{2}\right) x_{i}^{2}\right| \leqq 1 / 4$. Thus $\left|a_{0}\right| \geqq 2^{n / 2}$ which completes the proof. $2^{n}(x-1 / 2)^{n}$ shows that $\left|a_{0}\right| \geqq 2^{n / 2}$ is best possible.

Schur in his proof makes use of the fact that the discriminant of $f_{n}(x)$ has to be an integer. If we make use of this fact we easily obtain that, for large $n,\left|a_{0}\right|>\left(2^{1 / 2}+c\right)^{n}$. On p. 390 of his paper Schur constructs a polynomial of degree $2 n$ with

$$
a_{0}^{(2 n)}=\frac{1}{2(2)^{1 / 2}}\left(\left(1+2^{1 / 2}\right)^{n+1}-\left(1-2^{1 / 2}\right)^{n+1}\right) .
$$

${ }^{4}$ Math. Zeit. vol. 1 (1918) pp. 377-402, see p. 389-391. 
This seems the greatest possible value of $\left|a_{0}^{(2 n)}\right|$.

In the same paper (Theorem XIII, pp. 397-398) Schur proves the following theorem: Let $a_{0}$ be a given integer, and let $f_{n}(z)$ $=a_{0} z^{n}+\cdots+a_{n}$ be a polynomial with integer coefficients the roots of which either all have absolute value 1 and are different or all are in the interior of the unit circle (in which case multiple roots are permitted). Denote these roots by $z_{1}, z_{2} \cdots, z_{n}$. Then

$$
\lim \sup \frac{z_{1}+z_{2}+\cdots+z_{n}}{n} \leqq 1-\frac{e^{1 / 2}}{2} .
$$

Schur conjectures that the limit (6) is 0 , and remarks that if $a_{0}=1$ this follows from a theorem of Kronecker, which asserts that in this case all the $z_{i}$ are roots of unity. We now prove Schur's conjecture.

THEOREM 4. Let the $z_{i}$ be defined as above. Then

$$
\lim \frac{z_{1}+z_{2}+\cdots+z_{n}}{n}=0 .
$$

First we can assume that $n$ tends to infinity (that is, for every $n$ there are only a finite number of equations satisfying the conditions of the theorem). Also if $f(z)$ has all its root in the interior of the unit circle then $z^{2} f(z)+z^{n} f\left(z^{-1}\right)=g(z)$ has all its roots on the unit circle and all are different. Also the sum of the roots of $f(z)$ and $g(z)$ are identical (p. 397). Thus it will suffice to consider polynomials having all their roots on the unit circle and distinct.

Therefore the discriminant of $D$ satisfies the inequality

$$
1 \leqq D=a_{0}^{2 n-2} \prod_{i<j \leqq n}\left(z_{i}-z_{j}\right)^{2}
$$

It follows from a result of Pólya (p. 395) that

$$
\left|f\left(z_{1}, z_{2}, \cdots, z_{n}\right)\right|=\prod_{i<j \leqq n}\left(z_{i}-z_{j}\right) \leqq n^{n} .
$$

Thus for at least one $z$

$$
\prod_{j \neq i}\left|\left(z_{i}-z_{j}\right)\right| \leqq n
$$

To prove Theorem 4 it clearly suffices to show that the $z_{i}$ are uniformly distributed on the unit circle. Suppose this is not true. Then it follows from a result of Fekete $^{5}$ that there exists a $z_{0},\left|z_{0}\right|=1$, such that

${ }^{5}$ Ann. of Math. vol. 41 (1940) pp. 162-173, see pp. 165-166. 


$$
\prod_{j \neq i}\left|\left(z_{0}-z_{j}\right)\right|>\left(1+c_{1}\right)^{n} .
$$

But then from (9) and (10)

$$
\mid f\left(z_{0}, z_{1}, \cdots, z_{i-1}, z_{i+1} \cdots z_{n}\right)>\frac{\left(1+c_{1}\right)^{n}}{n} f\left(z_{1}, z_{2}, \cdots, z_{n}\right) .
$$

If $z_{1}, z_{2}, \cdots, z_{n}$ are not uniformly distributed we can continue this process $c_{2} n$ times, and thus obtain $y_{1}, y_{2}, \cdots, y_{n},\left|y_{i}\right|=1$, so that

$$
\left|f\left(y_{1}, y_{2}, \cdots, y_{n}\right)\right|>\frac{\left(1+c_{1}\right)^{c_{2} n^{2}}}{n^{c_{2} n}}\left|f\left(z_{1}, z_{2}, \cdots, z_{n}\right)\right| .
$$

But from (7) and (11) we obtain

$$
\left|f\left(y_{1}, y_{2}, \cdots, y_{n}\right)\right|>\left(1+c_{3}\right)^{n^{2}} \frac{1}{a_{0}^{2 n-2}}>n^{n}
$$

which contradicts (8) and completes the proof of Theorem 4.

Szegö ${ }^{6}$ proved the following theorem: Let $M$ be any closed set in the plane. Denote by $\omega_{n}\left(M, z_{0}\right)$ the maximum of $\left|f_{n}^{\prime}\left(z_{0}\right)\right|$ for all polynomials $f_{n}(z)$ of degree $n$ which satisfy $\left|f_{n}(z)\right| \leqq 1$ for all $z$ in $M$. Assume that the transfinite diameter of $M$ is positive. Then

$$
\lim \omega_{n}\left(M, z_{0}\right)^{1 / n}<\infty .
$$

Fekete ${ }^{7}$ proved that if $z_{0}$ is not in $M, \lim \omega_{n}\left(M, z_{0}\right)^{1 / n}$ exists and is finite if the transfinite diameter of $M$ is positive, and is infinite if the transfinite diameter of $M$ is 0 .

Assume now that $z_{0}$ belongs to $M$. The following questions remain open: (1) Does $\lim \omega_{n}\left(M, z_{0}\right)^{1 / n}$ exist? (2) Let the transfinite diameter of $M$ be 0. Is $\lim \omega_{n}\left(M, z_{0}\right)^{1 / n}=\infty$ ??

We are going to answer both questions in the negative. In fact we prove the following theorem.

THEOREM 5. Let $M$ be the set consisting of 0 and $1 / 2^{k}, k=0,1,2, \ldots$ Then

$$
\omega_{n}(M, 0)<c^{n} .
$$

Clearly $M$ is closed and countable, thus its transfinite diameter is 0 .

Lemma. Let $a, b, d$ be three real numbers, $d-b=b-a$. Then if $\left|f_{n}(z)\right|<1$ for $a<z<b$,

${ }^{6}$ Math. Zeit. vol. 23 (1925) pp. 45-61.

7 Math. Zeit. vol. 26 (1927) pp. 324-344. 


$$
f_{n}^{\prime}(d)<c_{1}^{n} /(b-a) .
$$

If $a=0, b=1$ the lemma follows from Szegö's ${ }^{6}$ result. The general case follows by a linear transformation. As a matter of fact it is well known that $\omega_{n}(M, d)$ is given in this case by the Tchebychef polynomial belonging to $(a, b)$.

Now we prove Theorem 5 . The equation $f_{n}^{2}(z)=1$ can have at most $2 n$ real roots. Thus since $\left.\mid f(1 / 2)^{k}\right) \mid<1, k=0,1, \cdots$, we obtain that, for some $k>n+1,\left|f_{n}(z)\right|<1$ for all $1 / 2^{k}<z<1 / 2^{k+1}$. Thus by the lemma

$$
\left|f_{n}^{\prime}(0)\right|<2^{n+1} c_{1}^{n}<c^{n}
$$

q.e.d.

Theorem 6. Let the set $M$ be defined as follows: $n_{1}<n_{2}<\cdots$ tend to infinity sufficiently fast. $M$ consists of the points 0 and $1 / 2^{u}$ where $n_{i} \leqq u \leqq 2 n_{i}+1$. Then $\lim \omega_{n}(M, 0)^{1 / n}$ does not exist. In fact $\lim \sup \omega_{n}(M, 0)^{1 / n}=\infty, \lim \inf \omega_{n}\left(M, z_{0}\right)^{1 / n}<\infty$.

As in Theorem 5 it follows that if $\left|f\left(1 / 2^{u}\right)\right|<1$ for $n_{i} \leqq u \leqq 2 n_{i}+1$, $f(x)$ a polynomial of degree $n_{i}$, then $\omega_{n_{i}}(M, 0)<c^{n_{i}}$. Consider $f(x)$ $=2^{n_{i}+1} \prod\left(x-1 / 2^{k}\right), k \leqq 1,2, \cdots, 2 n_{i}+1$. The degree of $f(x)$ equals $2 n_{i}+2$. Also $|f(x)|<1$ for all $x$ in $M$, and if $n_{i+1}$ tends to infinity sufficiently fast

$$
\left(f^{\prime}(0)\right)^{1 / 2 n_{i}+2}>\left(2^{n_{i}+1} / 2^{\left(2 n_{i}+1\right)^{2}}\right)^{1 / 2 n_{i}+2} \rightarrow \infty
$$

q.e.d.

THEOREM 7. Let $f_{n}(z)$ be a polynomial of degree $n$ with real coefficients. $\left|f_{n}(z)\right|<1$ for $-1 \leqq z \leqq 1$. Then if $\left|z_{0}\right| \geqq 1$

$$
\left|f_{n}\left(z_{0}\right)\right| \leqq\left|T_{n}\left(z_{0}\right)\right| \text {. }
$$

Equality holds only for $f_{n}(z)= \pm T(z)$.

In case $z_{0}$ is real this result is well known.

We are going to prove the following more general result: Let $\left|f_{n}\left(x_{i}\right)\right| \leqq 1$ where $x_{1}=-1, x_{i}, i=1,2, \cdots, n-1$, are the roots of $T_{n}^{\prime}(x)$ and $x_{n}=1$. Then for $\left|z_{0}\right| \geqq 1$

$$
\left|f_{n}\left(z_{0}\right)\right| \leqq\left|T_{n}\left(z_{0}\right)\right| \text {. }
$$

We have $\left(l_{i}(x)=\omega(x) / \omega^{\prime}\left(x_{i}\right)\left(x-x_{i}\right), \omega(x)=\left(1-x^{2}\right) T_{n}^{\prime}(x)\right)$

$$
f_{n}\left(z_{0}\right)=\sum_{i=0}^{n} y_{i} l_{i}\left(x_{0}\right), \quad\left|y_{i}\right| \leqq 1, y_{i} \text { real. }
$$


We evidently have for complex numbers $A$ and $B, \max (|A+B|$, $|A-B|>A$. Thus $\left|f_{n}(z)\right|$ will be maximal if $y_{i}= \pm 1$. A simple geometric argument shows that the angle between any two of the vectors $(-1) i l_{i}\left(z_{0}\right)$ is less than $\pi / 2$ (since the interval $(-1,+1)$ subtends from $z_{0}$ at an angle not greater than $\pi / 2$ ). But then clearly $\left|f_{n}\left(z_{0}\right)\right|$ is maximal if

$$
f_{n}\left(z_{0}\right)= \pm \sum_{i=0}^{n}(-1)^{i} l_{i}\left(z_{0}\right)= \pm T_{n}\left(z_{0}\right)
$$

Equality clearly occurs only if $f(z)= \pm T(z)$.

Corollary. Let $\left|f_{n}(z)\right| \leqq 1$ for $-1 \leqq z \leqq 1$, also let $f_{n}(z)$ have real coefficients. Then for $|z| \leqq 1,\left|f_{n}(z)\right|<\left|T_{n}(i)\right|$.

If we do not assume that the coefficients of $f_{n}(z)$ are real it is easy to give examples which show that $\left|f_{n}(z)\right|$ does not have to be less than $\left|T_{n}(i)\right|$. Trivially $\left|f_{n}(z)\right| \leqq \sum_{k=0}^{n}\left|l_{k}(i)\right|$. But in general $\max \left|f_{n}(z)\right|<\sum_{k=0}^{n}\left|l_{k}(i)\right|$. I can not at present determine $\max \left|f_{n}(z)\right|$ for $|z| \leqq 1$.

In the same way we can prove that if $f(z)=a_{0} z^{n}+\cdots+a_{n}$ has real coefficients and $|f(z)| \leqq 1$ for $-1 \leqq z \leqq 1$ then $\sum_{k=0}^{n}\left|a_{k}\right|$ is maximal for $f(z)= \pm T_{n}(z)$. Szegö ${ }^{8}$ proved the following stronger result: $\left|a_{2 k}\right|+\left|a_{2 k+1}\right|$ is maximal for $f(z)= \pm T_{n}(z)$.

Syracuse UnIversity

${ }^{8}$ Oral communication. 